\title{
A REMARK ON DOMAINS QUASICONFORMALLY EQUIVALENT TO A BALL
}

\author{
F. W. GEHRING *
}

1. Introduction. An important problem in the theory of higher dimensional quasiconformal mappings is to characterize geometrically those domains $D$ in $\overline{R^{n}}$, the one point compactification of euclidean $n$ space $R^{n}$, which can be mapped quasiconformally onto the unit ball $B^{n}$ in $R^{n}$. The following rather implicit criterion appears to be the best result known in this direction at present [6].

Th e o r e m 1. Suppose that $D$ is a domain in $\bar{R}^{n}$ and suppose that for some neighborhood $U$ of $\partial D$ there exists a quasiconformal mapping $f$ of $D \cap U$ into $B^{n}$ such that $|f(x)| \rightarrow 1$ as $x \rightarrow \partial D$ in $D \cap U$. Then $D$ is quasiconformally equivalent to $B^{n}$.

Theorem 1 can be localized as follows for the case when $D$ is a Jordan domain, that is, when $\partial D$ is homeomorphic to $\partial B^{n},[6]$ and [13].

Th e o r e m 2. Suppose that $D$ is a Jordan domain in $\bar{R}^{3}$ and suppose that for each $x \in \partial D$ there exists a neighborhood $U$ of $x$ and a quasiconformal mapping $f$ of $D \cap U$ into $B^{3}$ such that $|f(y)| \rightarrow 1$ as $y \rightarrow \partial D$ in $D \cap U$. Then $D$ is quasiconformally equivalent to $B^{3}$.

It seems likely that Theorem 2 is still true with 3 replaced by $n$ throughout. However the proof given in [6] depends crucially on Ahlfors' affirmative solution, when $n=2$, of the lifting conjecture for quasiconformal mappings [1].

C o n j e c t u r e. Each quasiconformal mapping $f: R^{n} \rightarrow R^{n}$ can be extended to a quasiconformal mapping $g: R^{n+1} \rightarrow R^{n+1}$.

Carleson has recently established this conjecture for $n=3$ [4], and his proof suggests a method to settle the conjecture for all $n \geqq 4$.

If we apply Theorem 2 and Theorem 17.12 of [15], we obtain a simple geometric condition sufficient to guarantee that a domain $D$ in $R^{3}$ is quasiconformally equivalent to $B^{3}$.

* This research was supported in part by the U.S. National Science Foundation, Grant MPS 7507940. 
Cor ollary 1. Suppose that $D$ is a bounded Jordan domain in $R^{3}$, that $D$ has a tangent plane $T$ at each point of $\partial D$, and that $T$ is continuous in $\partial D$. Then $D$ is quasiconformally equivalent to $B^{3}$.

Unfortunately the example given below shows that this sufficient condition is very far from being necessary. (See also [7].)

Example 1 . For each $n \geqq 3$ there exists a domain $D$ in $R^{n}$ such that $D$ is quasiconformally equivalent to $B^{n}$ and such that $D$ does not have a tangent plane at any point of $\partial D$.

Proof. Let

$$
H=\left\{x=\left(x_{1}, \ldots, x_{n}\right): x_{n}<0\right\},
$$

let $\partial H$ denote the usual imbedding of $\bar{R}^{n-1}$ in $\bar{R}^{n}$, and choose a sequence of open $(n-1)$-balls $B_{j}$ in $R^{n-1}$ so that the $\bar{B}_{j}$ are pairwise disjoint and so that $\cup_{j} B_{j}$ is dense in $R^{n-1}$. Next set

$$
D=H \cup\left(\bigcup_{j} P^{-1}\left(B_{j}\right)\right),
$$

where $P$ denotes orthogonal projection of $R^{n}$ into $R^{n-1}$. Then the argument in [8] or on page 462 of [3] shows that $D$ is quasiconformally equivalent to $B^{n}$, while the fact that $D$ is dense in $R^{n}$ implies that $D$ does not have a tangent plane at any point of its boundary.

On the other hand the following example shows that the geometric hypotheses in Corollary 1 cannot be weakened significantly. (See also [11].)

$\mathrm{E} \times \mathrm{am} \mathrm{p} 1 \mathrm{e} 2$. For each $n \geqq 3$ there exists a bounded Jordan domain $D$ in $R^{n}$ such that $D$ has a tangent plane $T$ at each point of $\partial D$, such that $T$ is continuous at all but one point of $\partial D$, and such that $D$ is not quasiconformally equivalent to $B^{n}$.

Examples 1 and 2 show that there is no satisfactory way to characterize the domains $D$ in $R^{n}$ which are quasiconformally equivalent to $B^{n}$ in terms of their tangential properties.

The purpose of this note is to establish the existence of the domain mentioned in Example 2. The proof depends on an inequality, given in Section 3 , between the $(n-1)$-modulus of a family of curves in the boundary and the $n$-modulus of a corresponding family in the interior of a ball or half space.

2. Moduli of curve families. For $q \in[0, \infty)$ the normalized $q$ dimensional Hausdorff outer measure of a set $E$ in $R^{n}$ is defined by

$$
\mathscr{H}^{q}(E)=\lim _{t \rightarrow 0}\left(\inf \sum_{j} \alpha(q) 2^{-q} \operatorname{dia}\left(E_{j}\right)^{q}\right),
$$


where the infimum is taken over all countable coverings of $E$ by sets $E$, with dia $\left(E_{j}\right)<t$ and where

$$
\alpha(q)=\frac{\Gamma\left(\frac{1}{2}\right)^{q}}{\Gamma\left(\frac{q}{2}+1\right)} .
$$

If $q$ is an integer in $[1, n]$ and $T$ a $q$-dimensional hyperplane in $R^{n}$, then $\mathscr{H}^{q} \mid T$ coincides with the Lebesgue $q$-dimensional outer measure $m_{q}$ in $T$.

For $p, q \in[1, \infty)$ the $q$-dimensional $p$-modulus of a curve family $\Gamma$ in $\overline{R^{n}}$ is defined as

$$
M_{p}^{q}(\Gamma)=\inf \int_{R^{n}} h^{p} d \mathscr{H}^{q},
$$

where the infimum is taken over all $h$ in $\operatorname{adm}(\Gamma)$, the collection of all Borel functions $h: R^{n} \rightarrow[0, \infty]$ such that $\int_{\gamma} h d s \geqq 1$ for all locally rectifiable curves $\gamma$ in $\Gamma$. One often writes $M_{p}(\Gamma)$ for $M_{p}^{n}(\Gamma)$ and $M(\Gamma)$ for $M_{n}^{n}(\Gamma)$.

We will need a symmetry principle for the moduli of curve families, namely the $n$-dimensional analogue of Lemma 3.3 in [8]. We take the opportunity to establish here the following more general result by means of a somewhat different argument.

$\mathrm{L} \mathrm{e} \mathrm{m} \mathrm{m} \mathrm{a} \mathrm{1.} \mathrm{Suppose} \mathrm{that} D$ is an open half space in $R^{n}$, that $E$ and $F$ are disjoint compact sets in $\bar{D}$, and that $E^{*}$ and $F^{*}$ are the symmetric images of $E$ and $F$ in $\partial D$. If $\Gamma_{1}$ and $\Gamma_{2}$ are the families of curves joining $E$ to $F$ in $D$ and $E \cup E^{*}$ to $F \cup F^{*}$ in $\bar{R}^{n}$, respectively, then

$$
M_{p}^{n}\left(\Gamma_{2}\right)=2 M_{p}^{n}\left(\Gamma_{1}\right) \text {. }
$$

The proof depends upon the following result.

L e $\mathrm{m} \mathrm{m}$ a 2. Suppose that $D, E, F, \Gamma_{1}$ are as in Lemma 1 and that $\Gamma$ is the family of curves joining $E$ to $F$ in $\bar{D}$. Then

$$
M_{p}^{n}(\Gamma)=M_{p}^{n}\left(\Gamma_{1}\right) \text {. }
$$

Proof. Since $\Gamma_{1} \subset \Gamma$, it is sufficient to prove that

$$
M_{p}^{n}(\Gamma) \leqq M_{p}^{n}\left(\Gamma_{1}\right) \text {. }
$$

Let $e$ denote the inner unit normal for $\partial D$, choose $t \in(0,1)$, and set

$$
f(x)=x+t \operatorname{dist}(x, E \cup F) e
$$

for $x \in R^{n}$ and $f(\infty)=\infty$. Then $f$ is a homeomorphism of $\bar{R}^{n}$ onto itself and 


$$
(1-t)|x-y| \leqq|f(x)-f(y)| \leqq(1+t)|x-y|
$$

for all $x, y \in R^{n}$.

Choose $g \in \operatorname{adm}\left(\Gamma_{1}\right)$ and set $h=(1+t) g \circ f$ in $R^{n}$. Then each locally rectifiable curve $\gamma$ in $\Gamma$ contains a subcurve $\gamma^{\prime}$ which $f$ maps into $\Gamma_{1}$ and

$$
\int_{\gamma} h d s \geqq \int_{\gamma^{\prime}}(1+t) g \circ f d s \geqq \int_{f\left(\gamma^{\prime}\right)} g d s \geqq 1
$$

by (5). Thus $h \in \operatorname{adm}(\Gamma)$ and

$$
M_{p}^{n}(\Gamma) \leqq \int_{R^{n}} h^{p} d m_{n} \leqq \frac{(1+t)^{p}}{(1-t)^{n}} \int_{R^{n}} g^{p} d m_{n} .
$$

Taking the infimum over all such $g$ gives

$$
M_{p}^{n}(\Gamma) \leqq \frac{(1+t)^{p}}{(1-t)^{n}} M_{p}^{n}\left(\Gamma_{1}\right),
$$

and we obtain (4) by letting $t \rightarrow 0$.

Proof for Lemma 1. Let $r: \bar{R}^{n} \rightarrow \bar{R}^{n}$ denote reflection in $\partial D$. Then $\Gamma_{1}$ and $r\left(\Gamma_{1}\right)$ are separate subfamilies of $\Gamma_{2}$ and

$$
M_{p}^{n}\left(\Gamma_{2}\right) \geqq M_{p}^{n}\left(\Gamma_{1}\right)+M_{p}^{n}\left(r\left(\Gamma_{1}\right)\right)=2 M_{p}^{n}\left(\Gamma_{1}\right) .
$$

Next let $\Gamma$ be as in Lemma 2, choose $g \in \operatorname{adm}(\Gamma)$, and set $h=g \circ f$ in $R^{n}$, where

$$
f(x)= \begin{cases}x & \text { if } x \in \bar{D} \\ r(x) & \text { if } x \in r(\bar{D})\end{cases}
$$

If $\gamma$ is a locally rectifiable curve in $\Gamma_{2}$, then $f(\gamma) \in \Gamma$ and

$$
\int_{\gamma} h d s \geqq \int_{f(\gamma)} g d s \geqq 1
$$

Hence $h \in \operatorname{adm}\left(\Gamma_{2}\right)$,

$$
M_{p}^{n}\left(\Gamma_{2}\right) \leqq \int_{R^{n}} h^{p} d m_{n}=\int_{D} g^{p} d m_{n}+\int_{r(D)}(g \circ r)^{p} d m_{n} \leqq 2 \int_{R^{n}} g^{p} d m_{n},
$$

and taking the infimum over all such $g$ yields

$$
M_{p}^{n}\left(\Gamma_{2}\right) \leqq 2 M_{p}^{n}(\Gamma) .
$$

The desired conclusion then follows from (3), (6) and (7). 
3. An inequality. We establish next an inequality between the moduli of two families of curves joining the same pair of disjoint continua.

L e m m a 3. Suppose that $2 \leqq m<n<\infty$, that $R^{m}$ is the usual imbedding of euclidean $m$-space in $R^{n}$, and that $E$ and $F$ are nondegenerate disjoint continua in $\bar{R}^{m}$. If $\Gamma_{\mathbf{1}}$ and $\Gamma_{\mathbf{2}}$ are the families of curves which join $E$ to $F$ in $\bar{R}^{m}$ and $\bar{R}^{n}$, respectively, then

$$
-2 n<\left(\frac{M_{m}^{m}\left(\Gamma_{1}\right)}{\beta(m)}\right)^{1 /(1-m)}-\left(\frac{M_{n}^{n}\left(\Gamma_{2}\right)}{\beta(n)}\right)^{1 /(1-n)}<2 m,
$$

where $\beta(q)=q \alpha(q)$ and $\alpha(q)$ is as in (1).

Proof. By performing a preliminary Möbius transformation, we may assume that $0 \in E$ and that $\infty \in F$. Arguing as in Lemma 3.5 of [8], we can find continua $C_{0}$ and $C_{1}$ in $\bar{R}^{m}$ such that $\partial C_{0} \subset E \subset C_{0}, \partial C_{1} \subset$ $F \subset C_{1}$ and such that $R=\bar{R}^{m} \sim\left(C_{0} \cup C_{1}\right)$ is an $m$-dimensional ring domain. Then $S=\bar{R}^{n} \sim(E \cup F)$ is an $n$-dimensional ring domain,

$$
\operatorname{cap}_{m}(R)=M_{m}^{m}\left(\Gamma_{1}\right), \quad \operatorname{cap}_{n}(S)=M_{n}^{n}\left(\Gamma_{2}\right)
$$

and (8) is equivalent to the inequality

$$
-2 n<\bmod _{m}(R)-\bmod _{n}(S)<2 m .
$$

To establish (9) set $a=\sup \{|x|: x \in E\}, b=\inf \{|x|: x \in F\}$. Then by a well known estimate for the moduli of rings,

$$
\bmod _{m}(R) \leqq \log \lambda_{m}^{2}\left(\frac{b}{a}+1\right)
$$

where $\lambda_{m}$ is a constant which depends only on $m$. (See [3], [5], [12] and [14].) If $a<b$, then $E$ and $F$ are separated in $\overline{R^{n}}$ by the spherical ring

$$
T=\left\{x \in R^{n}: a<|x|<b\right\} .
$$

In this case,

$$
\log \frac{b}{a}=\bmod _{n}(T) \leqq \bmod _{n}(S)
$$

and we obtain

$$
\bmod _{m}(R)-\bmod _{n}(S)<\log 2 \lambda_{m}^{2} .
$$

If $a \geqq b$, then (10) follows trivially since $\bmod _{n}(S)>0$. From estimates in [2] it follows that $\log 2 \lambda_{m}^{2}<2 m$, and we obtain the second half of (9) from (10). The first half follows from interchanging the roles of $m$ and $n$ in the above argument. 
$\mathrm{R}$ e m a rk. The hypothesis that $E$ and $F$ be connected is essential in Lemma 3.

Proof. Let $h:(0,1) \rightarrow(0, \infty)$ denote the Hausdorff measure function $h(t)=(\log (1 / t))^{1-n}$. Then

$$
\lim _{t \rightarrow 0} h(t)\left(\log \frac{1}{t}\right)^{m-1}=0 .
$$

By Theorem 4.4 in [16], there exists a compact linear set $E$ in the unit ball $B^{m} \subset R^{m}$ such that $E$ is of positive conformal capacity as a subset of $R^{m}$ and such that $E$ is of Hausdorff $h$-measure zero. By Theorem 4.3 in [16], this last fact implies that $E$ is of zero conformal capacity as a subset of $R^{n}$.

Let $F=\bar{R}^{m} \sim B^{m}$, let $\Gamma_{1}$ and $\Gamma_{2}$ be as in Lemma 3 , and let $\Gamma_{2}^{\prime}$ denote the family of curves joining $E$ to $F^{\prime}=\bar{R}^{n} \sim B^{n}$ in $\bar{R}^{n}$. Then each $\gamma$ in $\Gamma_{2}$ contains a subcurve $\gamma^{\prime}$ in $\Gamma_{2}^{\prime}$, and

$$
M_{m}^{m}\left(\Gamma_{1}\right)>0, \quad M_{n}^{n}\left(\Gamma_{2}\right) \leqq M_{n}^{n}\left(\Gamma_{2}^{\prime}\right)=0
$$

by known results relating extremal length and conformal capacity. (See, for example, [10].) Hence inequality (8) cannot hold in this case.

Corollary 2. Suppose that $n \geqq 3$, that $D$ is an open ball or half space in $R^{n}$, and that for $j=1,2, \ldots, E_{j}$ and $F_{j}$ are pairs of nondegenerate disjoint continua in $\partial D$. If $\Gamma_{1, j}$ and $\Gamma_{2, j}$ are the families of curves joining $E_{j}$ to $F_{j}$ in $\partial D$ and in $D$, respectively, and if either

$$
\lim _{j \rightarrow \infty} M_{n-1}^{n-1}\left(\Gamma_{1, j}\right)=0 \quad \text { or } \quad \lim _{j \rightarrow \infty} M_{n}^{n}\left(\Gamma_{2, j}\right)=0,
$$

then

$$
\lim _{j \rightarrow \infty} \frac{M_{n-1}^{n-1}\left(\Gamma_{1, j}\right)^{(n-1) /(n-2)}}{M_{n}^{n}\left(\Gamma_{2, j}\right)}=c,
$$

where $c$ is a positive constant which depends only on $n$.

Proof. By performing a preliminary Möbius transformation, we may assume that

$$
D=\left\{x=\left(x_{1}, \ldots, x_{n}\right): x_{n}>0\right\}
$$

and that $\partial D$ is the usual imbedding of $\bar{R}^{n-1}$ in $\bar{R}^{n}$. If $\Gamma_{3, j}$ denotes the family of curves joining $E_{j}$ to $F_{j}$ in $\bar{R}^{n}$, then

by Lemma 1. Hence

$$
M_{n}^{n}\left(\Gamma_{3, j}\right)=2 M_{n}^{n}\left(\Gamma_{2, j}\right)
$$

$$
\left|\left(\frac{M_{n-1}^{n-1}\left(\Gamma_{1, j}\right)}{\beta(n-1)}\right)^{1 /(2-n)}-\left(\frac{2 M_{n}^{n}\left(\Gamma_{2, j}\right)}{\beta(n)}\right)^{1 /(1-n)}\right|<2 n
$$


by Lemma 3, and (11) implies (12) with

$$
c=2 \beta(n-1)^{(n-1) /(n-2)} \beta(n)^{-1} .
$$

4. Proof for Example 2. For $j=1,2, \ldots$, set $a_{j}=4^{-j}$ and define $f:[0, \infty) \rightarrow(-1,0]$ by

$$
f(t)=\left\{\begin{array}{l}
a_{j}^{2}\left(\cos \left(\pi a_{j}^{-3} t\right)-1\right) \text { if } t \in\left(a_{j}, 2 a_{j}\right) \text { for some } j, \\
0 \text { otherwise. }
\end{array}\right.
$$

Then $f$ is continuously differentiable in $(0, \infty),|f(t)| \leqq 2 t^{2}$ in $[0, \infty)$, and

for all $j$. Let

$$
1 \leqq \int_{a_{j}}^{2 a_{j}}\left|f^{\prime}(t)\right| d t \leqq \pi
$$

$$
D=\left\{x=\left(x_{1}, \ldots, x_{n}\right): x_{n}>f(r)\right\}, \quad r=\left(x_{1}^{2}+\cdots+x_{n-1}^{2}\right)^{1 / 2},
$$

and let $g: \bar{R}^{n} \rightarrow \bar{R}^{n}$ be any Möbius transformation for which $g^{-1}(\infty) \notin \bar{D}$. Then $g(D)$ is a bounded Jordan domain, $g(D)$ has a tangent plane $T$ at each point of its boundary, and $T$ is continuous except at the single point $g(0)$.

To complete the proof it suffices to show that $D$, and hence $g(D)$, is not quasiconformally equivalent to $B^{n}$. For each $j$ let

$$
S_{j}=\left\{x \in \partial D: r \in\left(a_{j}, 2 a_{j}\right)\right\}, \quad E_{j}=\left\{x \in \partial D: r \in\left[2 a_{j}, 4 a_{j}\right]\right\},
$$

where $r$ is as in (14). Next let $\Gamma_{1, j}$ and $\Gamma_{2, j}$ denote the families of curves which join $E_{j}$ to $E_{j+1}$ in $\partial D$ and in $D$, respectively.

To obtain an upper bound for $M_{n-1}^{n-1}\left(\Gamma_{1, j}\right)$ set $h$ equal to the characteristic function of $S_{j}$. If $\gamma$ is a locally rectifiable curve in $\Gamma_{1, j}$, then $\gamma$ contains a subcurve $\gamma^{\prime}$ which lies, except for its endpoints, in $S_{j}$. Hence with (13) we have

$$
\int_{\gamma} h d s \geqq \int_{\gamma^{\prime}} d s \geqq \int_{a_{j}}^{2 a_{j}}\left|f^{\prime}(t)\right| d t \geqq 1,
$$

$h \in \operatorname{adm}\left(\Gamma_{1, j}\right)$, and

$$
\begin{aligned}
M_{n-1}^{n-1}\left(\Gamma_{1, j}\right) & \leqq \int_{S_{j}} d \mathscr{H}^{n-1} \\
& \leqq \beta(n-1) \int_{a_{j}}^{2 a_{j}} r^{n-2}\left(\left|f^{\prime}(r)\right|+1\right) d r \leqq c_{1} a_{j}^{n-2},
\end{aligned}
$$

where $c_{1}$ is a finite constant which depends only on $n$. 
To obtain a lower bound for $M_{n}^{n}\left(\Gamma_{2, j}\right)$ let

$$
H=\left\{x \in\left(x_{1}, \ldots, x_{n}\right): x_{n}>0\right\} .
$$

Then $E_{j} \subset \partial H$ and $H \subset D$. Let $\Gamma_{3, j}$ denote the family of curves which join $E_{j}$ to $E_{j+1}$ in $H$. Then $\Gamma_{2, j} \supset \Gamma_{3, j}$, and since the Möbius transformation $k(x)=4^{j-1} x$ maps $\Gamma_{3, j}$ onto $\Gamma_{3,1}$, we obtain

$$
M_{n}^{n}\left(\Gamma_{2, j}\right) \geqq M_{n}^{n}\left(\Gamma_{3, j}\right)=M_{n}^{n}\left(\Gamma_{3,1}\right)=c_{2},
$$

where $c_{2}$ is a positive constant which depends only on $n$.

Now suppose there exists a $K$-quasiconformal mapping $h$ which maps $D$ onto a ball $D^{\prime}$. Then $h$ has a homeomorphic extension $h^{*}$ which maps $\partial D$ onto $\partial D^{\prime}$. Let $\Gamma_{4, j}$ denote the family of curves which join $E_{j}$ to $E_{j+1}$ in $S_{j}$, and let $\Gamma_{i, j}^{\prime}$ denote the image of $\Gamma_{i, j}$ under $h^{*}$. Since $h$ is $K$-quasiconformal in $D$,

$$
M_{n}^{n}\left(\Gamma_{2, j}\right) \leqq K M_{n}^{n}\left(\Gamma_{2, j}^{\prime}\right)
$$

Next since $D$ has a continuous tangent plane at each point of $S_{j}, S_{j}$ is an admissible boundary surface for $D$ and

$$
M_{n-1}^{n-1}\left(\Gamma_{4, j}^{\prime}\right) \leqq K M_{n-1}^{n-1}\left(\Gamma_{4, j}\right)
$$

by Theorem 2.23 in [9]. (See also [3] where (18) is derived with the factor $K$ replaced by $K^{n-2}$.) Since $\Gamma_{4, j} \subset \Gamma_{1, j}$ and since each curve in $\Gamma_{1}$, contains a subcurve in $\Gamma_{4, j},(18)$ implies that

$$
M_{n-1}^{n-1}\left(\Gamma_{1, j}^{\prime}\right) \leqq K M_{n-1}^{n-1}\left(\Gamma_{1, j}\right) \text {. }
$$

But (15), (16), (17) and (19) imply that

$$
\lim _{j \rightarrow \infty} M_{n-1}^{n-1}\left(\Gamma_{1, j}^{\prime}\right)=0, \quad \lim _{j \rightarrow \infty} \frac{M_{n-1}^{n-1}\left(\Gamma_{1, j}^{\prime}\right)^{(n-1) /(n-2)}}{M_{n}^{n}\left(\Gamma_{2, j}^{\prime}\right)}=0,
$$

contradicting the conclusion of Corollary 2. Thus $D$ is not quasiconformally equivalent to a ball, and the proof is complete.

\section{References}

[1] AhrFORS, L. V.: Extension of quasiconformal mappings from two to three dimensions. - Proc. Nat. Acad. Sci. USA 51, 1964, 768- 771.

[2] Anderson, G. D.: Dependence on dimension of a constant related to the Grötzsch ring. - Proc. Amer. Math. Soc. (to appear).

[3] Caraman, P.: $n$-Dimensional quasiconformal (QCf) mappings. - Editura Academiei București / Abacus Press, Tunbridge Wells, Kent, 1974.

[4] CARLESON, L.: The extension problem for quasiconformal mappings. - Contributions to analysis: A collection of papers dedicated to Lipman Bers, Academic Press, New York-London, 1974, 39-47. 
[5] GEHRING, F. W.: Symmetrization of rings in space. - Trans. Amer. Math. Soc. 101, 1961, 499-519.

[6] -»- Extension theorems for quasiconformal mappings in $n$-space. - J. Analyse Math. 19, 1967, 149-169.

[7] GeHring, F. W., and B. P. PALKA: Quasiconformally homogeneous domains. J. Analyse Math. (to appear).

[8] GEHRING, F. W., and J. VÄISÄL $\ddot{A}$ : The coefficients of quasiconformality of domains in space. - Acta Math. 114, 1965, 1-70.

[9] HAG, K.: Quasiconformal boundary correspondences and extremal mappings. Ph.D. thesis, University of Michigan, 1972.

[10] HESSE, J.: A $p$-extremal length and $p$-capacity equality. - Ark. Mat. 13, 1975, $131-144$.

[11] Kopylov, А. Р. [А. П. Копылов]: О степени гладкости границы одласти, гомеоморфной шару и не являющейся квазиконформным одразом шара. - Sibirsk. Mat. Ž. 11, 1970, 1181-1183. Translation: The degree of smoothness of the boundary of a region homeomorphic to a ball without being the quasiconformal image of a ball. - Siberian Math. J. $11,1970,877-878$.

[12] MosTow, G. D.: Quasi-conformal mappings in $n$-space and the rigidity of hyperbolic space forms. - Inst. Hautes Études Sci. Publ. Math. 34, 1968, 53-104.

[13] NäkkI, R.: Boundary behavior of quasiconformal mappings in $n$-space. Ann. Acad. Sci. Fenn. Ser. A I 484, 1970, 1-50.

[14] Sarvas, J.: Symmetrization of condensers in $n$-space. - Ann. Acad. Sci. Fenn. Ser. A I 522, 1972, 1-44.

[15] VÄISÄL̈̈, J.: Lectures on $n$-dimensional quasiconformal mappings. - Lecture Notes in Mathematics 229, Springer-Verlag, Berlin-HeidelbergNew York, 1971.

[16] WALLIN, H.: Metrical characterization of conformal capacity zero. - J. Math. Anal. Appl. (to appear).

University of Michigan

Department of Mathematics

Ann Arbor, Michigan 48109

USA

Received 20 December 1975

\section{University of Paris}

Department of Mathematics

F-91405 Orsay

France 